\section{The evolving role of exercise in cancer patients: recent developments, recommendations and future directions 2016}
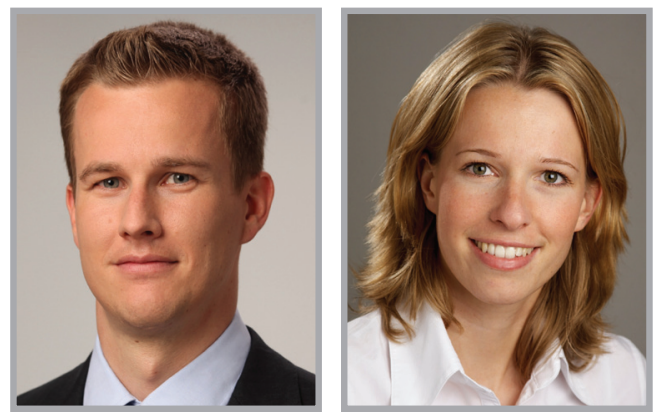

\author{
"Research into the \\ combination of exercise and \\ anticancer therapy would also \\ help in integrating exercise \\ programs and counseling into \\ standard clinical care.”
}

Joachim Wiskemann ${ }^{*, 1}$ \& Friederike Scharhag-Rosenberger ${ }^{1}$

First draft submitted: 28 January 2016; Accepted for publication: 3 March 2016; Published online: 29 March 2016
Although 'exercise and cancer' is a relatively new area of research, more than 100 randomized controlled exercise intervention trials (RCTs) have already been published [1] and the interest of researchers, clinicians, nurses, exercise therapist, funding agencies and patients is still growing. Considering this rapid development it is worth reflecting on previous findings and addressing future directions.

The 'exercise and cancer' story began in the early 1980s with retrospective epidemiological observations like those of Garabrant et al. [2] showing that occupational physical activity is associated with a reduced risk for colon cancer. Today, a large number of observational studies demonstrate associations between all kinds of physical activity and reduced cancer risk in nearly all common cancer entities except for rectal cancer [3]. With regard to less common cancer entities the knowledge is insufficient or nonexisting. Overall, it is estimated that between 9 and 19\% of cancer cases (of the major cancer sites) are attributable to insufficient physical activity [3].

Building on this knowledge of primary prevention, further cohort studies focused on tertiary prevention, in other words, associations between physical activity and cancer specific as well as overall survival. A recent meta-analysis summarized findings of a total of 49,095 breast and colorectal cancer survivors and discovered an impressive relative risk reduction through physical activity of approximately $48 \%$ in breast cancer and $42 \%$ in colorectal cancer survivors when comparing the highest versus the lowest postdiagnosis physical activity level in each study [4]. Prediagnosis physical activity was also inversely associated with postdiagnosis mortality. In prostate cancer survivors, a reduction in mortality of up to $61 \%$ was observed in patients who performed more than $90 \mathrm{~min}$ of vigorous

\section{KEYWORDS}

- oncology • physical activity

- physical fitness $\bullet$ survival $\bullet$ training
'Department of Medical Oncology, National Center for Tumor Diseases (NCT) \& Heidelberg University Hospital, Heidelberg, Germany

*Author for correspondence: Tel.: +49 622156 5904; Fax: +49 622156 7225; joachim.wiskemann@nct-heidelberg.de 
“...one major goal of all efforts done in the past, present and future is to perform high-quality trials with regard to efficacy, efficiency and implementation possibilities to convince insurance companies to cover costs for exercise therapy in cancer patients." physical activity/week compared with predominantly inactive patients [5]. It should be noted that the minimal amount of physical activity needed to benefit was relatively high in all analyses and, interestingly, it was twice as high for colorectal cancer patients compared with breast cancer patients (18 vs 9 metabolic equivalent task.h/week) [4].

The latest observational studies focus on the prediction of survival based on physical performance or fitness assessments. In non-small-cell lung cancer patients a simple 6 -min walk test proved to be highly predictive of mortality, showing a $56 \%$ risk reduction for walk distances above $400 \mathrm{~m}$ compared with distances below $400 \mathrm{~m}$ [6]. Similarly peak oxygen uptake $\left(\mathrm{VO}_{2 \text { peak }}\right)$ derived from cardiopulmonary exercise tests is a strong independent predictor of deaths in surgical candidates with non-small-cell lung cancer [7] or women with metastatic breast cancer $[8] . \mathrm{VO}_{2 \text { peak }}$ and six-minute walk distance were predictors of symptom severity, quality of life and mortality [9] in allogeneic stem cell transplant patients. Therefore increases in physical performance or fitness appear desirable and it is evident from numerous RCTs that this can be achieved through regular exercise training.

One major flaw of the aforementioned observational studies is that it cannot be ruled out that the inverse association between physical activity or physical fitness and mortality reflects reversed causation, meaning that patients who feel better perform better. This situation has changed in 2014 when Courneya et al. published follow-up data from an exercise intervention RCT in 242 breast cancer patients undergoing chemotherapy [10]. They found beneficial but nonsignificant effects of resistance and endurance training on disease-free survival, overall survival and distant disease-free survival after a median follow-up of 89 months [10]. Also a median follow-up of 61 months of a 12-week aerobic exercise intervention study during primary treatment in lymphoma patients suggests beneficial effects of exercise on progression-free survival [11]. Furthermore, data of our group demonstrate a significantly reduced 2-year total mortality and nonrelapse mortality in allogeneic stem cell transplant patients who exercised prior, during and up to 8 weeks after transplantation [12]. These three intervention studies recently raised the scientific knowledge to a new level but there is still one major limitation: no study reported exercise training data for the follow-up period and therefore, improved survival might be attributable to other factors. To overcome this limitation there are currently two ongoing RCTs trying to confirm the above reported findings and consistently report exercise training data until follow-up $[12,13]$.

These latest findings enhanced the interest in potential mechanistic links between exercise training and survival. Beneficial changes in insulin, inflammation and possibly hormone oxidative stress as well as immune-related pathways have been discussed for some years [14]. Furthermore, few studies report impressive effects of exercise training during chemotherapy on completion rates [15]. Combined with the observation that higher chemotherapy completion rates may improve disease-free and overall survival [16], these findings may have important clinical implications. Closely related to these are discussions on the potential effect of exercise to mitigate cardiotoxicity of chemotherapy. The focus on serious complications and treatment completion rates may introduce a new area of research that combines exercise with pharmacological approaches.

Research into the combination of exercise and anticancer therapy would also help in integrating exercise programs and counseling into standard clinical care. Patients would clearly benefit from this development since there is convincing evidence from numerous RCTs of positive effects of exercise training on various treatment side effects and cancer-related problems within the physical and psychosocial domain [17]. This holds true for curatively treated patients where positive effects on, for example, physical fitness, fatigue and quality of life are well documented. In contrast, only few studies exist in the palliative situation and benefits of physical activity are by far less clear. For example, resistance training might increase muscle mass and reduce cachexia in theory, but studies specifically in cachectic patients do not exist. Therefore, further research on the effects of exercise in palliative cancer patients is needed before recommendations for this specific population can be derived.

An important further step in the field of 'exercise and cancer' addresses the translation from scientific findings into practice: until now, the optimal type, frequency, duration and intensity of exercise training for cancer patients remain largely unknown. When analyzing previous RCTs, the above mentioned characteristics of exercise training were often either not prescribed or not reported [18]. Furthermore, adequate 
reporting of adherence to the training program is lacking in a couple of investigations resulting in vague information on effective exercise recommendations. Recent studies for the first time acknowledged these and some other difficulties such as differences in intensity prescription between cancer patients and healthy populations [19]. Further studies are strongly needed to optimize exercise prescription and until then, recommendations will remain rather general and similar to that for the general population adapted on the basis of the individual health status.

Considering the integration of exercise into standard clinical care, the most effective way might be a side effects-oriented exercise therapy pathway approach, which includes an initial patient screening with regard to existing symptoms or treatments that are closely linked with symptoms that can be addressed by exercise [20]. This would lead to some kind of standard operation procedures that can be integrated into clinical decision making systems. As a consequence, physicians would be able to individually assign patients to exercise programs. Such a systematic approach can be expanded to other supportive care services such as psycho-oncology, nutritional counseling and social services. A regular bio-psycho-social tumor board may help to structure the supportive care services with regard to patients' individual needs.

One main barrier with regard to these recommendations might be that the costs for exercise therapy are mostly not covered by insurance companies or restricted to a very short period of rehabilitation (far) beyond finishing primary treatment. Therefore, one major goal of all efforts done in the past, present and future is to perform high-quality trials with regard to efficacy, efficiency and implementation possibilities to convince insurance companies to cover costs for exercise therapy in cancer patients.

\section{Financial \& competing interests disclosure}

The authors have no relevant affiliations or financial involvement with any organization or entity with a financial interest in or financial conflict with the subject matter or materials discussed in the manuscript. This includes employment, consultancies, honoraria, stock ownership or options, expert testimony, grants or patents received or pending, or royalties.

No writing assistance was utilized in the production of this manuscript.

\section{References}

1 Battaglini CL, Mills RC, Phillips BL et al. Twenty-five years of research on the effects of exercise training in breast cancer survivors: A systematic review of the literature. World J. Clin. Oncol. 5(2), 177-190 (2014).

2 Garabrant DH, Peters JM, Mack TM, Bernstein L. Job activity and colon cancer risk. Am. J. Epidemiol. 119(6), 1005-1014 (1984).

3 Friedenreich CM, Neilson HK, Lynch BM. State of the epidemiological evidence on physical activity and cancer prevention. Eur. J. Cancer 46(14), 2593-2604 (2010).

4 Schmid D, Leitzmann MF. Association between physical activity and mortality among breast cancer and colorectal cancer survivors: a systematic review and metaanalysis. Ann. Oncol. 25(7), 1293-1311 (2014).

5 Kenfield SA, Stampfer MJ, Giovannucci E, Chan JM. Physical activity and survival after prostate cancer diagnosis in the health professionals follow-up study. J. Clin. Oncol. 29(6), 726-732 (2011).

6 Kasymjanova G, Correa JA, Kreisman $\mathrm{H}$ et al. Prognostic value of the six-minute walk in advanced non-small cell lung cancer. J. Thorac. Oncol. 4(5), 602-607 (2009).

7 Jones LW, Watson D, Herndon JE et al. Peak oxygen consumption and long-term all-cause mortality in nonsmall cell lung cancer. Cancer 116(20), 4825-4832 (2010).

8 Jones LW, Courneya KS, Mackey JR et al. Cardiopulmonary function and age-related decline across the breast cancer survivorship continuum. J. Clin. Oncol. 30(20), 2530-2537 (2012).

9 Wood WA, Deal AM, Reeve BB et al. Cardiopulmonary fitness in patients undergoing hematopoietic SCT: a pilot study. Bone Marrow Transplant. 48(10), 1342-1349 (2013).

10 Courneya KS, Segal RJ, Mckenzie DC et al. Effects of exercise during adjuvant chemotherapy on breast cancer outcomes. Med. Sci. Sports Exerc. 46(9), 1744-1751 (2014).

11 Courneya KS, Friedenreich CM, FrancoVillalobos $\mathrm{C}$ et al. Effects of supervised exercise on progression-free survival in lymphoma patients: an exploratory follow-up of the HELP Trial. Cancer Causes Control 26(2), 269-276 (2015).

12 Wiskemann J, Kleindienst N, Kuehl R, Dreger P, Schwerdtfeger R, Bohus M. Effects of physical exercise on survival after allogeneic stem cell transplantation. Int. J. Cancer 137(11), 2749-2756 (2015).

13 Courneya KS, Booth CM, Gill S et al. The Colon Health and Life-Long Exercise Change trial: a randomized trial of the National Cancer Institute of Canada Clinical Trials Group. Curr. Oncol. 15(6), 271-278 (2008).

14 Ballard-Barbash R, Friedenreich CM, Courneya KS, Siddiqi SM, Mctiernan A, Alfano CM. Physical activity, biomarkers, and disease outcomes in cancer survivors: a systematic review. J. Natl Cancer Inst. 104(11), 815-840 (2012).

15 Van Waart H, Stuiver MM, Van Harten WH et al. Effect of low-intensity physical activity and moderate- to high-intensity physical exercise during adjuvant chemotherapy on physical fitness, fatigue, and chemotherapy completion rates: results of the PACES randomized clinical trial. J. Clin. Oncol. 33(17), 1918-1927 (2015).

16 Early Breast Cancer Trialists' Collaborative G, Peto R, Davies C et al. Comparisons between different polychemotherapy regimens for early breast cancer: meta-analyses of long-term outcome among 100,000 women in 
EDITORIAL Wiskemann \& Scharhag-Rosenberger

123 randomised trials. Lancet 379(9814), 432-444 (2012).

17 Mishra SI, Scherer RW, Geigle PM et al. Exercise interventions on health-related quality of life for cancer survivors. Cochrane Database Syst. Rev. 8, CD007566 (2012).

18 Campbell KL, Neil SE, Winters-Stone KM. Review of exercise studies in breast cancer survivors: attention to principles of exercise training. Br. J. Sports Med. 46(13), 909-916 (2012).

19 Scharhag-Rosenberger F, Kuehl R, Klassen O et al. Exercise training intensity prescription in breast cancer survivors: validity of current practice and specific recommendations. J. Cancer Surviv. 9(4), 612-619 (2015).
20 Wiskemann J, Scharhag-Rosenberger F. [Side effect-oriented exercise therapy pathways of oncological patients].

Nebenwirkungsorientierte Behandlungspfade für die bewegungstherapeutische Betreuung onkologischer Patienten. Tumor Diagn. Ther. 36(2), 91-95 (2015). 